\title{
ANÁLISIS DE PROPUESTAS DE INNOVACIÓN EDUCATIVA EN EL PRÁCTICUM DEL GRADO EN PEDAGOGÍA
}

\author{
Esteban Vázquez-Cano \\ M. ${ }^{a}$ Luisa Sevillano García \\ Francisco De Pedro Sotelo \\ Universidad Nacional de Educación a Distancia
}

RESUMEN: En este artículo se presenta un estudio en el que se analizan las concepciones del alumnado universitario sobre la innovación educativa a partir de su proceso de trabajo en el prácticum de Pedagogía en la UNED. La muestra participante está constituida por 170 estudiantes pertenecientes a cuarto curso del Grado en Pedagogía durante el curso académico 2016/17. La investigación se ha desarrollado desde una doble vertiente cualitativa: análisis documental de la memoria final del trabajo de la asignatura y el análisis de foros de discusión de la plataforma informática de la UNED. Para ello, se ha empleado el programa Atlas-Ti y del enfoque reticular-categorial del análisis de redes sociales con la aplicación de UCINET. Los resultados más significativos muestran que los cinco ámbitos en los que los estudiantes han identificado y propuesto un mayor número de actuaciones son los siguientes: convivencia escolar $(\mathrm{n}=56)$, acoso $(n=93)$, formación del profesorado $(n=87)$, metodologías activas $(n=88)$ y educación en valores $(n=32)$. En este sentido, el estudio aporta una visión alternativa a la innovación a través de la mirada del alumno del último curso del Grado en Pedagógica como consecuencia de sus prácticas profesionales.

PALABRAS CLAVE: Innovación, educación, prácticas profesionales, pedagogía, prácticum.

\section{ANALYSIS OF EDUCATIONAL INNOVATION PROPOSALS IN THE PRACTICUM OF THE DEGREE IN PEDAGOGY}

\footnotetext{
ABSTRACT: In this article, a study is presented in which university students' conceptions about educational innovation are analyzed through their work in the Pedagogy Practicum at UNED. The participating sample consists of 170 students belonging to the fourth course of the Degree in Pedagogy during the 2016/17 academic year. The research has been developed from a double
} 
qualitative perspective: text analysis of the final report of the subject and the analysis of discussion forums of UNED digital platform. For this purpose, the use of the Atlas-Ti program and the reticular-categorial approach of social network analysis with the application of UCINET have been used. The most significant results show that the five areas in which students have identified and proposed a greater number of actions are the following: school coexistence $(n=56)$, harassment $(n=93)$, teacher training $(n=87)$, active methodologies $(n=88)$ and education in values $(n=32)$. In this sense, the study provides an alternative vision to innovation through the eyes of the student of the last year of the Degree in Pedagogy, as a result of their professional practices.

KEYWORDS: Innovation, education, internships, pedagogy, practicum.

Recibido: 18/02/2018

Aceptado: 11/07/2018

Correspondencia: Esteban Vázquez-Cano, Universidad Nacional de Educación a Distancia, C/ Juan del Rosal, 14, 28040 Madrid. Email: evazquez@edu.uned.es.

\section{INTRODUCCIÓN}

El desarrollo de las prácticas profesionales en el ámbito de la educación Ileva aparejado la visita y trabajo en centros educativos y formativos. En este periodo, el estudiante va conformando la realidad de la profesión como pedagogo y pone en práctica muchos de los conceptos teóricos vistos desde las asignaturas que conforman el Grado en Pedagogía; lo que le permitirá transformarlos en competencias profesionales. Ahí, desde nuestro punto de vista, reside el valor formativo del Prácticum. En este sentido, uno de los trabajos fundamentales del estudiante es intentar observar, analizar y proponer medidas de intervención que promuevan cambios e innovaciones que repercutan en un mejor funcionamiento de las instituciones formativas y educativas. Atendiendo a este principio, este estudio pretende analizar la actividad discente en la asignatura de "Prácticas Profesionales V" del Grado en Pedagogía de la UNED e identificar aquellos ámbitos que desde la perspectiva del estudiante de Pedagogía serían susceptibles de intervención para fomentar procesos de innovación educativa en la educación actual.

\section{La innovación educativa y el Prácticum}

La innovación educativa se inicia desde el cuestionamiento de las prácticas existentes y tiene como principal objetivo provocar cambios en los procesos de enseñanza aprendizaje que repercutan en la mejora de la Educación y de los agentes que intervienen en la misma (Fullan, 2002; Fundación Telefónica, 2016). Por lo tanto, el concepto de innovación no va asociado exclusivamente a la puesta práctica de actividades innovadoras, sino al resultado de las mismas (Sevillano García, Pascual Sevillano y Bartolomé Crespo, 2007; Vázquez-Cano, Sevillano, y Méndez, 2011). Asimismo, como manifiesta (Miranda Martín, 2002: p. 3): “El cambio educativo solo 
Ilega a ser significativo si activa los procesos de acción-reflexión-acción en los sujetos que lo llevan a cabo de forma participativa, cooperativa, negociada y deliberativa". Desde estos postulados, el cambio en la institución educativa puede venir provocado por planteamientos institucionales ampliamente consensuados y debatidos en el seno de los centros educativos, pero también desde la iniciativa individual de cualquier miembro de la comunidad educativa que inicia un proceso cuyos resultados positivos son posteriormente asumidos por la institución educativa.

En el marco del objetivo 4 de la Agenda 2030 para el Desarrollo sostenible, se establece un principio que asegure que todos los alumnos adquieran los conocimientos teóricos y prácticos necesarios para promover el desarrollo sostenible, mediante la educación. Con este horizonte el Prácticum ha adquirido en los planes de estudio de todos los grados una importancia significativa y la innovación dentro de los mismos, un carácter sustancial, atendiendo como se describe en la misma Agenda 2030 a la diversidad de los pueblos, culturas, costumbres, estilos de vida. Diferentes autores como Villar Angulo (2017: p. 48) reconoce que la preocupación por la innovación docente ha caracterizado la educación superior en todo el mundo. En su exposición da especial realce a comentar que las innovaciones en la enseñanza universitaria deben comprender la competencia del mercado en la sociedad y se lamenta que sean pocos los libros que traten sobre las competencias que precisan los profesores para idear, elaborar y poner en práctica innovaciones educativas.

Carlos Rosales (2017) de la Universidad de Santiago de Compostela y uno de los docentes que más ha reflexionado e investigado sobre innovación en Educación, dedica un capítulo en su libro a estudiar la imagen de la profesión docente (pp. 129165). Afirma en el apartado de métodos, técnicas y actividades que toda innovación pedagógica apuesta por superar las limitaciones de la metodología tradicional, fortaleciendo líneas de trabajo de carácter individualizado y socializado o cooperativo. En este caso, se potencia la comunicación y colaboración frente a la competitividad. En otra obra anterior (Rosales, 2012: p. 15) identifica y comenta características que la innovación de calidad debe reunir. Destacamos 5.

a) Los centros escolares pueden estimular la innovación en la medida en que hagan posible el desarrollo de una cultura emergente en la que se potencie la iniciativa de cada profesor y cada grupo. Una cultura de características innovadoras deberá fundamentarse en valores como el pluralismo (respeto a las personas, sus creencias, conceptos, posturas...), la apertura a la colaboración y el cambio, la objetividad en el reconocimiento de posibilidades y límites y la tolerancia con los propios errores al considerar la innovación como un proceso de medio o largo recorrido.

b) El desarrollo de la capacidad de innovación en el profesor y en los centros se vincula estrechamente con las características de la formación profesional docente inicial y, sobre todo, permanente. Es necesario que, desde el comienzo de su formación, tome contacto con conceptos y procesos innovadores. Pero es más importante aún que, a lo largo de su prolongada carrera profesional, el docente reciba estímulos y cuente con una serie de condiciones que hagan posible la tarea innovadora.

c) De manera específica, se vincula fuertemente con la innovación la capacidad de reflexión sobre la práctica, tanto a nivel individual como grupal. 
d) Cualquier iniciativa de innovación resultaría más viable en un clima de entendimiento y colaboración entre ambos.

e) La apertura del centro escolar a la comunidad constituye por si misma una manifestación de innovación de la enseñanza.

El proceso innovador no es un hecho individual y aislado, como tampoco lo es el aprendizaje. La innovación se desarrolla pues, en un contexto cultural determinado. Una agrupación de tal tipo es un ente social vivo, que tiene sus metas, imagen y proyección no coincidente con la suma de las individualidades. Determinados Centros escolares crecen y proyectan en su entorno una imagen atractiva para los padres y la administración. El prestigio o imagen pública de los centros de enseñanza viene dada, no solo por responder a las expectativas que de ellos se tiene, sino por su carácter innovador. Vázquez Cano (2017: p. 393), considera que en cualquier proceso y, más si cabe en Educación, precisa de mecanismos de investigación que permitan a los profesionales involucrados en las aulas, analizar si la didáctica, organización de aula, del centro y su evaluación, entre otros muchos aspectos, se pueden mejorar para promover un aprendizaje del alumnado más eficaz, competencial y útil para desenvolverse en cualquier cambio profesional, personal o académico. Más adelante (p. 419) puntualiza que la innovación educativa debe ser una tarea constante y un referente de la actuación educativa. La innovación educativa constituye uno de los principios sobre los que se asienta el sistema educativo. Se inicia desde el cuestionamiento de la práctica existente y tiene como objetivo principal provocar cambios en los procesos de enseñanza- aprendizaje que repercutan en la mejora de la educación y de los agentes que intervienen en la misma.

El concepto de innovación va por tanto asociado no solamente a la puesta en práctica de actividades innovadoras, sino a sus resultados. Por su parte González y Medina (2017) no duda en proponer la innovación continua como respuesta a los cambios que se producen en la sociedad, teniendo en cuenta que, en el hecho educativo diario, constituye un aspecto nuclear en la tarea educativa. Sin duda la competencia innovadora lleva al óptimo desempeño profesional del profesorado con el propósito de una elevada calidad en sus prácticas educativas. Podemos afirmar en consonancia con otros autores que la capacidad innovadora de los docentes tiene una gran trascendencia y va a permitir al futuro docente analizar la realidad educativa y diseñar propuestas de intervención pedagógica fundamentalmente innovadoras (Sevillano García, Pascual Sevillano y Bartolomé Crespo, 2007).

La innovación parte y se asienta en procesos bien diseñados, ordenados, secuenciados, verificables, evaluables, que se pueda saber en un plazo no muy largo de los resultados. La investigación ha de ser, el principio de la innovación, pero también de su puesta en práctica, su valoración, su crítica y su mejora de nuevo innovadora. La innovación busca un cambio cualitativo en las prácticas educativas y no solo mejoras puntuales. Entendemos que la innovación educativa, no solo ha de centrarse o buscar las grandes cuestiones, sino que deberá cooperar a la solución de problemas de la actividad diaria que la enseñanza-aprendizaje plantea a directivos, profesores, estudiantes y familias y darlas a conocer. Cuántas veces hemos observado cómo, conocido el problema, diagnosticadas las causas, avanzada la innovación, constatamos que otros también innovaron así. Es preciso crear una red de profesores y centros comprometidos en la innovación que ha de ser constante y permanente (OECD, 2014). 
La innovación como proceso y producto es una aportación creativa y valiosa que enriquece el bien cultural de una comunidad humana, que en nuestro caso sería la educativa. Diversas investigaciones analizadas (Atkinson, 2013; Christensen, 1997; López y Heredia, 2017) nos permiten inducir campos interesantes de innovación como los siguientes: El enfoque globalizador. La atención individualizada mediante procesos diferenciados dentro del aula. La utilización de métodos y recursos variados que potencien la creación y el uso de las nuevas estrategias propias de búsqueda y organización de los elementos requeridos para resolver un problema. La valoración de la investigación, la exploración y la búsqueda de solución por parte del alumno. La posibilidad del uso de diversas fuentes de información y recursos metodológicos. El fomento de la autonomía en la búsqueda de soluciones (procesos autónomos de autoaprendizaje). La generación de planes de trabajo y su revisión sistemática, tanto por parte del alumno como del profesor. La creación de un clima de aceptación mutua y la cooperación dinámica de grupos. La integración de medios didácticos variados como: recursos impresos, material para la realización de experiencias, medios audiovisuales, computador, etc.

El profesor Santos Rego y un grupo de profesores de las Universidades de Navarra, Córdoba, Valencia, Complutense y Santiago de Compostela (2017) han propuesto el "Diseño y validación de un cuestionario sobre práctica docente y actitud del profesorado universitario hacia la innovación". A las prácticas docentes innovadoras dedicaron 11 ítems de los 32 del cuestionario. Según sus resultados hay un primer factor que aglutina seis ítems que se refieren a las actividades innovadoras más centradas en los alumnos. Son resolución de problemas, promotora se participación, aprendizaje autónomo, aprendizaje permanente, desarrollo de capacidad crítica y actualización metodológica. Un segundo factor aglutina aquellos ítems relacionados con las actividades innovadoras centradas en la interacción con el medio, como trabajo interdisciplinar, liderazgo, empleabilidad, sensibilidad hacia temas medioambientales.

Este proceso de innovación entronca con las competencias pretendidas en el desarrollo de la asignatura de Prácticas Profesionales V en el Grado de Pedagogía de la UNED. Entre las competencias genéricas se destacan las siguientes: gestionar y planificar la actividad profesional, gestionar los procesos de mejora, calidad e innovación, trabajar en equipo, desarrollar actitudes éticas de acuerdo con los principios deontológicos y el compromiso social, promover actitudes acordes a los derechos humanos y los principios democráticos. Y como, competencias específicas, las siguientes:

- Comprender los referentes teóricos, históricos, socioculturales, político-comparados, ambientales y legales que constituyen al ser humano como protagonista de la educación.

- Diseñar planes, programas, proyectos, acciones y recursos adaptados a los distintos niveles del sistema educativo, en las modalidades presenciales y virtuales.

- Diseñar planes de formación del profesorado, de formadores y de otros profesionales, adecuados a las nuevas situaciones, necesidades y contextos.

- Evaluar políticas, instituciones y sistemas educativos.

- Supervisar centros, planes, programas y profesionales de la educación y la formación. 
- Asesorar sobre el uso pedagógico e integración curricular de los medios didácticos.

- Analizar, diseñar y evaluar las aplicaciones de las tecnologías de la información y la comunicación asociadas a los procesos educativos y formativos.

- Realizar estudios prospectivos y evaluativos sobre características, necesidades y demandas pedagógicas.

Por lo tanto, los principales objetivos de este estudio fueron los siguientes:

1. Analizar los trabajos de los estudiantes para identificar ámbitos de innovación educativa en la praxis profesional de los docentes desde la mirada del alumno universitario.

2. Proponer ámbitos de actuación encaminados a innovar en las aulas del Siglo XXI.

\section{Método}

La muestra participante está constituida por 170 estudiantes del cuarto curso del Grado de Pedagogía de la UNED que cursan la asignatura de Prácticas Profesionales. El $59,4 \%$ son alumnas $n=91$ y el $40,6 \%$ son alumnos $n=79$. El proceso de análisis se estructuró en dos fases de análisis; en primer lugar, recurrimos a un análisis cualitativo mediante identificación de frecuencias textuales más recurrentes en las memorias de los estudiantes correspondientes al trabajo final de la asignatura de Prácticum de cuarto curso del Grado de Pedagogía. En la fase primera, se realizó el filtrado en bruto de las memorias y trabajos de los estudiantes por medio de la técnica de "reducción de datos". Para ello, se categorizaron los textos y se codificaron los datos, identificando y diferenciando unidades de significado. Utilizamos para la codificación e interpretación de los datos el programa Atlas.Ti y recuento de frecuencias temáticas. En la fase segunda, se realizaron los procesos de "interpretación e inferencia".

En una segunda fase, se analizaron las intervenciones de los estudiantes en el foro de consultas, dudas y reflexiones de la asignatura desde la perspectiva del Análisis de Redes Sociales (ARS) para identificar las interacciones y las temáticas más relevantes en el desarrollo de los foros de discusión virtuales (Knoke y Yang, 2008). Este proceso es mucho más holístico que un método puramente cuantitativo y nos posibilita entender las propuestas de innovación educativa desde un entorno colaborativo e interpretativo nos permite (Caverlee, Liu y Webb, 2010). Hemos empleado en este análisis el programa UCINET 6 para el análisis reticular de la interacción comunicativa en los grupos de discusión y el visor yED Graph Editor 3.11.1 para editar el grafo resultante de la matriz de la red obtenida con UCINET y así poderlo hacer más comprensible. El esquema matricial que se ha empleado para la generación del grafo ha sido el siguiente:

$$
\operatorname{Pr}(\mathbf{Y}=\mathbf{y})=\left(\frac{1}{\kappa}\right) \exp \left\{\sum_{A} \eta_{A} g_{A}(\mathbf{y})\right\}
$$


Donde $\eta_{A}$ es el parámetro correspondiente a la configuración A (cuyo resultado no podrá ser cero si todos los pares de variables en A se asumen como "condicionalmente dependientes"). Asimismo, $g_{A}(y)=$ yij $\|_{A} y i j$ es la estadística de configuración de red que corresponde a $A ; g_{A}(y)=1$ si coincide con la observación de " $y$ " en la red, y si no parece en la red su resultado es $0 . \kappa$ es una cantidad que asegura que la normalización (1) es una adecuada distribución de probabilidad. Todos los modelos de grafos aleatorios exponenciales adoptan la forma Eq. (1) lo que implica una distribución de probabilidad general de los gráficos en " $n$ " nodos teniendo en cuenta que existen diferentes supuestos de dependencia que tienen la consecuencia de escoger diferentes tipos de configuraciones como relevantes para el modelo. Tomando en consideración esta ecuación, las únicas configuraciones que son relevantes para el modelo son aquellas en los que todos los lazos posibles en la configuración son mutuamente contingentes entre sí.

\section{RESULTADOS Y DISCUSIÓN}

La primera fase consistió en el análisis por parte de dos investigadores independientes de la base de datos textual de memorias de trabajo final de la asignatura Prácticas Profesionales de cuarto curso. Se estableció un primer análisis de las unidades textuales para medir la tendencia de intercódigos y solapamientos conceptuales. Para ello, se calculó el índice Guetzkow's U con el que se mide la fiabilidad del número de unidades identificadas por dos investigadores independientes, conforme a la siguiente fórmula (Holsti 1969):

$$
U=(\mathrm{O} 1-\mathrm{O} 2) /(\mathrm{O} 1+\mathrm{O} 2)
$$

O1 y O2 representan el número de unidades categorizadas respectivamente por cada uno de los investigadores que realizaron la codificación preliminar. Esta codificación supuso la identificación de 1678 unidades de significado agrupadas en 28 categorías deductivo-inductivas. Este análisis preliminar presentó un resultado en el índice Guetzkow's U de .0187, lo que representa casi un 88\% de coincidencia en el número de unidades identificadas por ambos investigadores. Asimismo, para verificar la consistencia interna de las unidades textuales identificadas se empleó el cotejo comparado de las unidades codificadas en el programa "excel". La consistencia fue alta con un $82,10 \%$ de coincidencia en el primer análisis, lo que se considera un índice alto (Simons, 1993; Weingart, Olekalns, y Smith, 2004). Posteriormente, se procedió a establecer el índice Kappa de Cohen para analizar la fiabilidad intercategorial. Para ello, empleamos el modelo sugerido por Brennan y Prediger (1981) calculado conforme a la siguiente fórmula:

$$
\kappa=\left(\Sigma \mathrm{P}_{\mathrm{ii}}-\Sigma \mathrm{P}_{\mathrm{i}} \times \mathrm{P}_{\mathrm{i}}\right) /\left(1-\Sigma \mathrm{P}_{\mathrm{i}} \times \mathrm{P}_{\mathrm{i}}\right)
$$

$\Sigma \mathrm{P}_{\mathrm{ii}}$ es el sumatorio de observaciones comunes y $\Sigma \mathrm{P}_{\mathrm{i}} \times \mathrm{P}_{\mathrm{i}}$ refleja la probabilidad de coincidencia (Holsti 1969). Los resultados obtenidos se presentan, en primer lugar, en la Tabla 1 donde se muestra la frecuencia de palabras asociadas a las temáticas del ámbito de la innovación educativa codificadas en el análisis de los trabajos de los estudiantes conforme a la categorización realizada por los dos investigadores. 
Tabla 1. Ámbitos de actuación para la innovación educativa

\begin{tabular}{lclc}
\hline $\begin{array}{c}\text { Ámbitos de innovación } \\
\text { educativa }\end{array}$ & Frecuencia & \multicolumn{1}{c}{$\begin{array}{c}\text { Ámbitos de innovación } \\
\text { educativa }\end{array}$} & Frecuencia \\
\hline Convivencia & 56 & Modelos de evaluación & 23 \\
\hline Adaptación de TIC a NEE & 13 & Bilingüismo & 27 \\
\hline Acoso escolar & 93 & Organización escolar & 8 \\
\hline Biblioteca & 4 & Voluntariado & 4 \\
\hline Ciberacoso & 51 & Cálculo mental & 9 \\
\hline Dinamización del patio escolar & 13 & Actividades extraescolares & 31 \\
\hline Formación del profesorado & 87 & Campeonatos & 10 \\
\hline Actividades deportivas & 7 & Familias & 34 \\
\hline Metodologías activas & 88 & Entorno & 21 \\
\hline Trabajo cooperativo & 34 & Mediación & 13 \\
\hline Desarrollo de competencias & 28 & Tutoría & 21 \\
\hline Ludificación & 21 & Deberes & 6 \\
\hline Huerto & 4 & Educación en valores & 32 \\
\hline Cinefórum & 1 & Abandono escolar & 17 \\
\hline
\end{tabular}

Como se puede observar en la Figura 1, los cinco ámbitos sobre los que se proponen innovaciones educativas son: convivencia escolar, acoso, formación del profesorado, metodologías activas y educación en valores. Estas temáticas también constituyen un referente en la innovación en otros estudios (Mendieta, Cobos, y Vázquez-Cano, 2016).

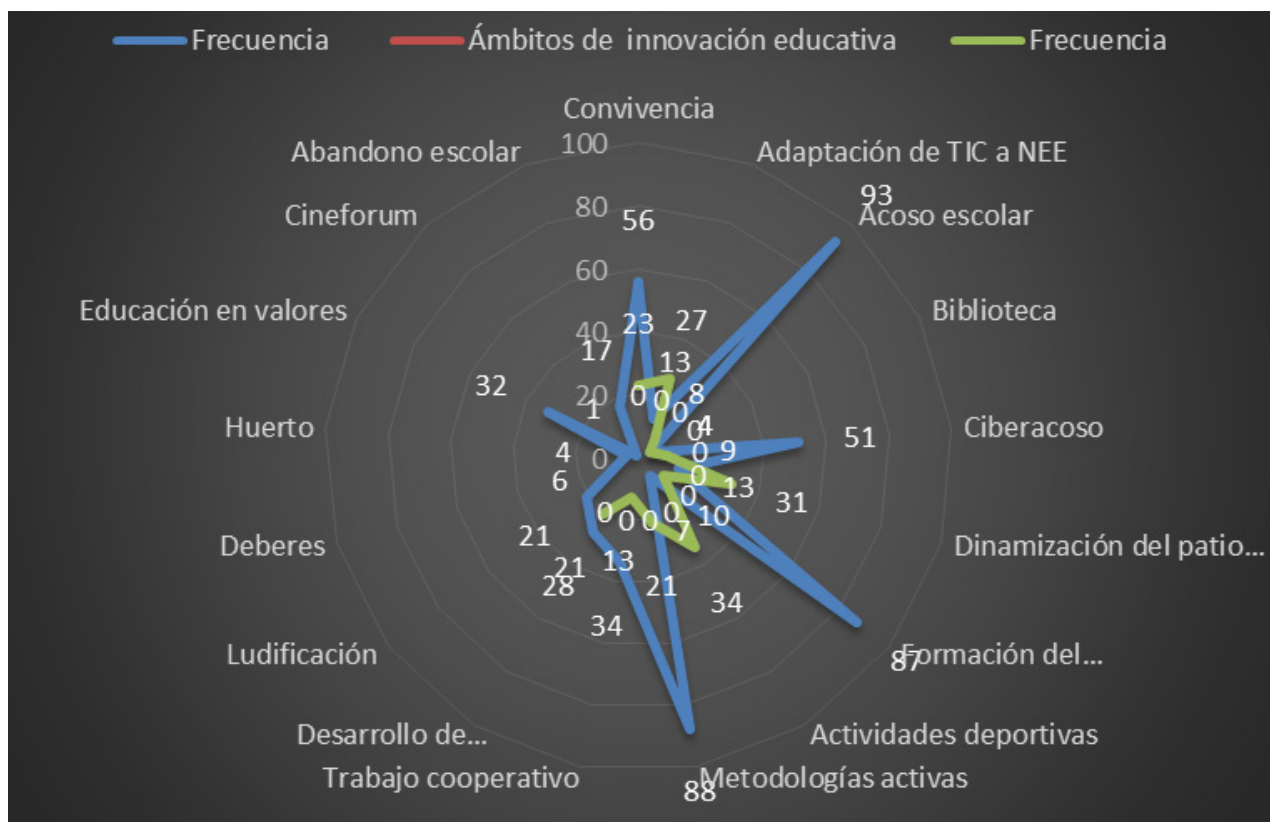

Figura 1. Frecuencia de aparición y su relación entre los ámbitos de innovación educativa 
A continuación, presentamos la red de Atlas-Ti con las relaciones establecidas entre las macrocategorías asociadas a la innovación y las innovaciones propiamente dichas. Como se puede observar en la Figura 2, la formación del profesorado es una macrocategoría transversal relacionada con las demás propuestas de innovación. Por lo tanto, es fundamental que para los procesos de innovación se arbitren procedimientos de formación del profesorado ágiles y versátiles que den respuestas a otros ámbitos de actuación de la innovación como son las metodologías activas, la gestión de la convivencia o problemas más puntuales como el acoso o el ciberacoso. Aspectos éstos que últimamente también están siendo propuestos en otros estudios desde la percepción del profesorado de diferentes etapas educativas (Castells y Monge, 2011; Escamilla, 2008; European Commission, 2007; Gimeno-Sacristán, 2008). Asimismo, se puede comprobar que la convivencia escolar también se ve afectada por el empleo de metodologías activas y el tratamiento de la educación en valores. La importancia de la educación en valores y su implicación en la convivencia escolar es otro elemento clave en el actual sistema educativo que viene siendo identificado por diversos estudios y autores (Bolívar, 2016; Calvo y Ballester, 2007; Vázquez-Cano, Sevillano, y Méndez, 2011).

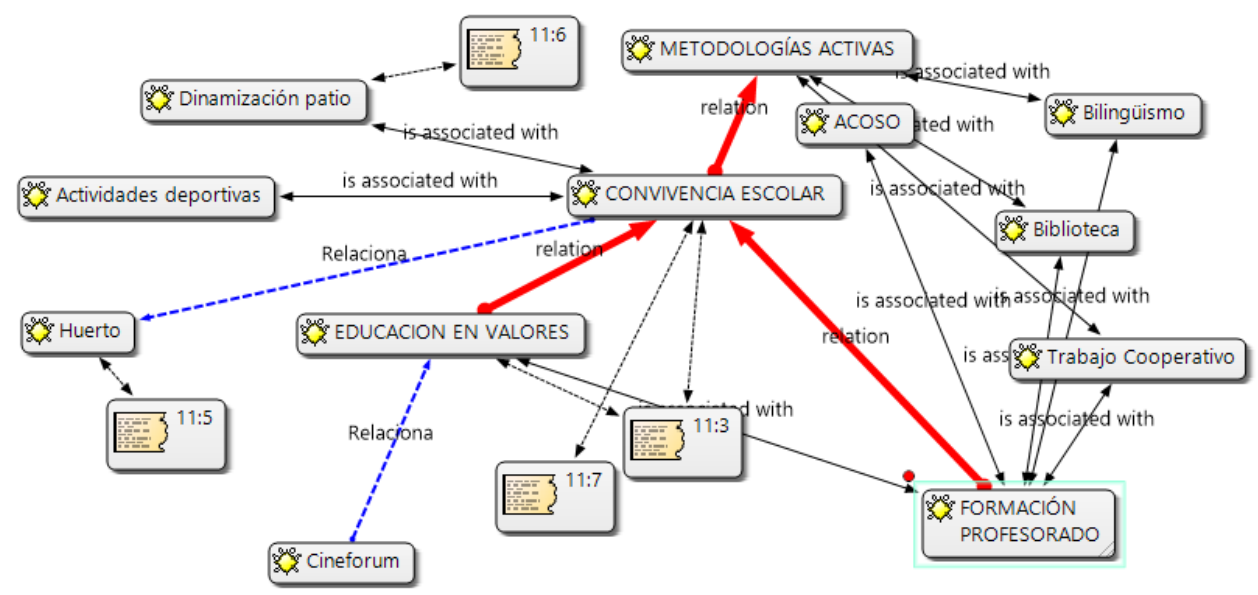

Figura 2. Red de relación en Atlas-Ti sobre la interrelación de categorías asociadas a la innovación

Con respecto a la innovación en metodologías activas, es importante reseñar que el desarrollo de procesos didácticos y metodológicos innovadores es uno de los mecanismos más efectivos para producir cambios en los procesos de enseñanzaaprendizaje, como se ha apuntado en recientes estudios e informes (Garavaglia, 2016; King y Baatartogtokh, 2015). La investigación y experimentación en nuevas formas de hacer más accesible, comprensivo y competencial el currículo es una 
de las principales competencias docentes. Los resultados de este estudio en este ámbito proponen cambios en la legislación y en el sistema educativo para generar nuevas miradas en didáctica y metodología que integren nuevos materiales y recursos, el desarrollo efectivo de las competencias claves con una aproximación más práctica en contextos y procesos que conjuguen el conocimiento teórico con una plasmación más funcional y práctica de los contenidos con aplicabilidad en la vida cotidiana como ya se ha propuesto en otros estudios (Narvekar, 2006; OECD, 2011).

El estudio de frecuencias textuales realizado con el programa Atlas-Ti se complementó con el análisis de las percepciones, comentarios y opiniones de los estudiantes de cuarto curso en los foros de la asignatura. Analizamos la red de interacciones y, para ello, hemos editado la red resultante de UCINET con el editor yED Graph Editor 3.11.1 (Figura 3) para hacerla más visual y comprensible.

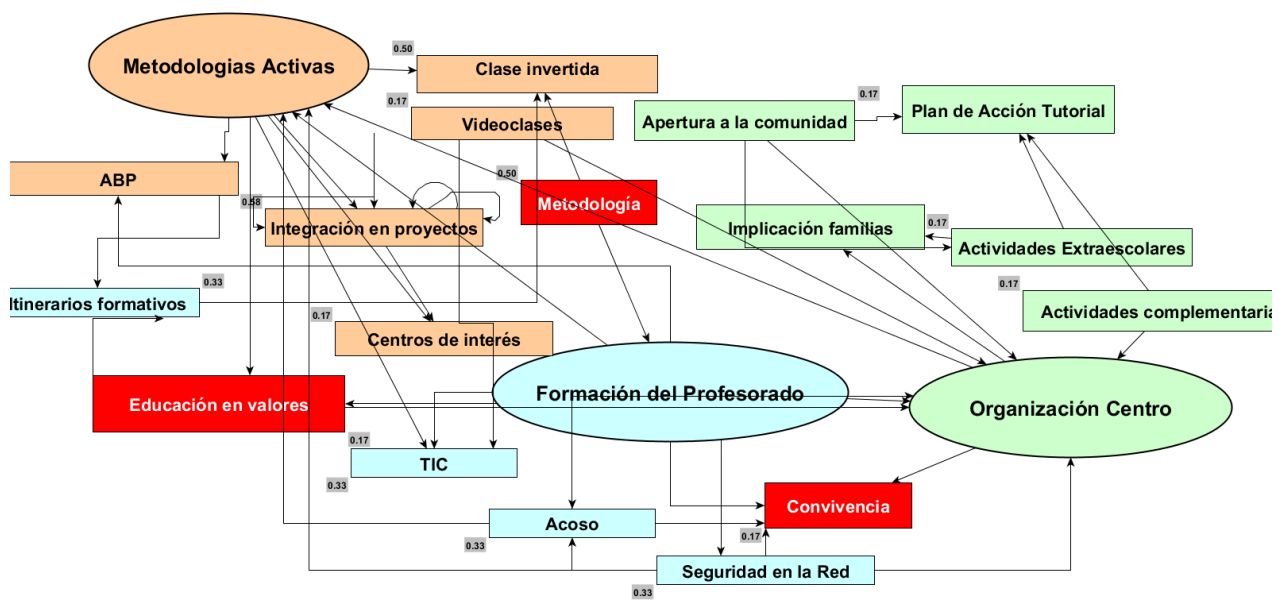

Figura 3. Red de innovación educativa en foros digitales

Podemos observar tres nodos principales en formato elipse ("Formación del profesorado", "Organización del centro" y "Metodologías activas") y tres colores para facilitar su identificación en la red. La densidad media del foro de discusión con la matriz dicotomizada fue .78 con .23 de desviación estándar; lo que representa un valor alto y un rango promedio de la red de 2.455; lo cual indica que cada palabra clave está interrelacionada con una media de casi 3. Además, este resultado muestra que más de dos tercios de todos los vínculos posibles estuvieron presentes y que se obtuvo una alta participación. Hemos analizado la centralidad de la red para identificar aquellos aspectos más prominentes. Para ello, hemos recurrido al análisis del grado nodal, de intermediación y de cercanía (Tabla 2). 
Tabla 2. Grado nodal, de intermediación y cercanía en la red "Innovación educativa"

\begin{tabular}{lcccccc}
\hline \multicolumn{1}{c}{ Ítems } & \multicolumn{2}{c}{$\begin{array}{c}\text { Grado } \\
\text { Nodal }\end{array}$} & \multicolumn{2}{c}{$\begin{array}{c}\text { Grado } \\
\text { Intermediación }\end{array}$} & \multicolumn{2}{c}{$\begin{array}{c}\text { Grado } \\
\text { Cercanía }\end{array}$} \\
\hline \multicolumn{1}{c}{ Formación del profesorado } & Deg. & NrmD. & Bet. & nBet. & Far. & Clos. \\
\hline TIC & 23.0 & 65.000 & 82.0 & 40.0 & 69.0 & 37.11 \\
\hline Acoso & 24.0 & 68.150 & 87.5 & 41.0 & 72.0 & 34.15 \\
\hline Seguridad en la red & 21.0 & 58.850 & 66.5 & 38.0 & 65.5 & 35.55 \\
\hline Itinerarios formativos & 23.0 & 61.150 & 81.5 & 39.5 & 71.5 & 30.55 \\
\hline \multicolumn{1}{c}{ Organización del centro } & Deg. & NrmD. & Bet. & nBet. & Far. & Clos. \\
\hline Apertura a la comunidad & 23.0 & 68.150 & 65.5 & 37.5 & 69.5 & 41.50 \\
\hline Plan de acción tutorial & 22.5 & 65.155 & 62.5 & 38.5 & 68.5 & 45.00 \\
\hline Implicación de las familias & 23.0 & 62.455 & 59.5 & 35.0 & 70.0 & 46.50 \\
\hline Actividades extraescolares & 19.0 & 44.550 & 40.5 & 21.5 & 50.5 & 32.50 \\
\hline Actividades complementarias & 20.5 & 46.110 & 40.0 & 19.5 & 48.0 & 31.10 \\
\hline \multicolumn{1}{c}{ Metodologías activas } & Deg. & NrmD. & Bet. & nBet. & Far. & Clos. \\
\hline Clase invertida & 23.0 & 60.120 & 55.5 & 30.0 & 61.0 & 41.10 \\
\hline Videoclases & 21.0 & 62.455 & 59.5 & 35.0 & 70.0 & 46.50 \\
\hline Integración de proyectos de centro & 22.0 & 61.450 & 59.5 & 35.0 & 70.0 & 46.50 \\
\hline Aprendizaje basado en proyectos & 24.0 & 65.450 & 62.5 & 38.5 & 68.5 & 45.00 \\
\hline Centros de interés & 22.0 & 60.550 & 58.5 & 37.5 & 68.0 & 42.00 \\
\hline
\end{tabular}

Como podemos observar los tres núcleos principales de innovación educativa se desglosan en 14 temáticas de discusión principal sobre la que giran buena parte de las intervenciones de los estudiantes en el foro. Destacan especialmente por su representación temática en los foros: la necesidad de formación del profesorado para mejorar el proceso y tratamiento del acoso escolar (Grado Nodal 24.0). Este resultado coindice con buena parte de la literatura científica que considera que la formación del profesorado es un elemento clave de muchos de los estudios e investigaciones que abogan por estrategias de innovación educativa (Cabero, 2004; Montero, 2006; Nieto, 2006). Asimismo, adquiere relevancia entre los estudiantes, la necesidad de innovar abriendo el centro educativo a la comunidad (Grado Nodal 23.0) e implicar en las familias en los procesos de enseñanza-aprendizaje formales y no formales de los estudiantes (Grado Nodal 23.0). La necesidad de innovar abriendo el centro educativo a la comunidad, principalmente desde el enfoque de comunidades de aprendizaje, es una de las propuestas que mejor resulta están teniendo en la conciencia socioeducativa hacia la innovación educativa (Coll, Bustos, y Engel, 2008; Delors, 1996).

Asimismo, se considera que es necesario formar la innovación desde la aplicación de metodologías activas, especialmente el aprendizaje basado en proyectos (Grado Nodal 24.0) y el desarrollo de la metodología de la clase invertida (Grado Nodal 23.0). Estas propuestas se han recogido profusamente en la literatura científica y, especialmente, en los informes Horizon (https://www.nmc.org/nmc-horizon/) y Edu- 
caTrends (https://observatorio.itesm.mx/acerca/). En este sentido y, analizadas las evidencias documentales y valoraciones realizadas en las memorias de trabajo de loe estudiantes y su participación en foros, mostramos, a continuación, algunos ejemplos de innovación educativa identificados y explicados en la memoria de los estudiantes.

Asimismo, los temas de convivencia y acoso se deben afrontar desde un sólido tratamiento de la educación en valores. La innovación en estrategias para fomentar y promover valores en la escuela y desarrollar proyectos que permitan a la comunidad educativa una sociedad más justa, respetuosa e igualitaria es uno de los ámbitos en los que más incidencia se puede realizar. En realidad, y, como no puede ser de otra forma, la Educación en Valores, como la educación en sí misma, no es un trabajo al que se pueda asignar un responsable, sino que debe ser la comunidad educativa al completo quien participe en su consecución. El contexto actual mundial y, particularmente la Unión Europea, deben hacer frente a una problemática común referente a la inmigración, la cohesión social y los diferentes conceptos morales que definan nuestros sistemas educativos. Es necesario conseguir que las ideas que nos unen y afectan a todos sean más fuertes que aquellas que nos separan y conseguir crear una conciencia democrática civil con el fin de prevenir los conflictos que vayan surgiendo en nuestro caminar diario. Podemos decir, con Antonio Bolívar (2016: 73), que:

Educar para el ejercicio de la ciudadanía supone primar la participación en todos los ámbitos escolares de todas las personas (incluidas las propias familias), como una comunidad que comparte por igual un conjunto de derechos democráticos de participación y comunicación. Además de la representación y participación de los distintos sectores en las instituciones escolares, el aprendizaje de la cultura democrática no acontece si no se dan otros procesos paralelos a generar desde la escuela y la comunidad.

En este sentido, una de las propuestas de intervención e innovación que realizan varios estudiantes están relacionados con los "talleres de mediación". Uno de los principales objetivos del Programa de Mediación es conseguir no ser solamente una herramienta de resolución de conflictos. Es importante conseguir el objetivo del diálogo, ser un elemento de diálogo, una nueva herramienta de capacitación del alumno y del equipo docente del centro escolar (Figura 4).

Por otro lado, la formación del profesorado es un ámbito esencial para favorecer la innovación educativa. Se considera fundamental que la preparación de los docentes vaya también encaminada a generar una cultura de trabajo y aprendizaje colaborativo y en red. La generación de redes permite que se pueda promover la creatividad, la flexibilidad y la agilidad del equipo docente ante los sucesivos retos del cambio social. En esta línea sería interesante que docentes jóvenes (nativos digitales) pudieran ejercer de mentores tecnológicos de docentes veteranos, y estos a su vez compartir sus estrategias de aula con los noveles. El segundo ámbito "refuerzo para el éxito educativo" pretende innovar para dar respuesta a los colectivos más vulnerables, en riesgo de exclusión socioeconómica y en etapas educativas en las que se intensifican los casos de fracaso y abandono escolar: desde $5^{\circ}$ de primaria a la ESO y Formación Profesional Básica. En este sentido los alumnos han propuesto talleres formativos en servicio para el profesorado (Figura 5). 


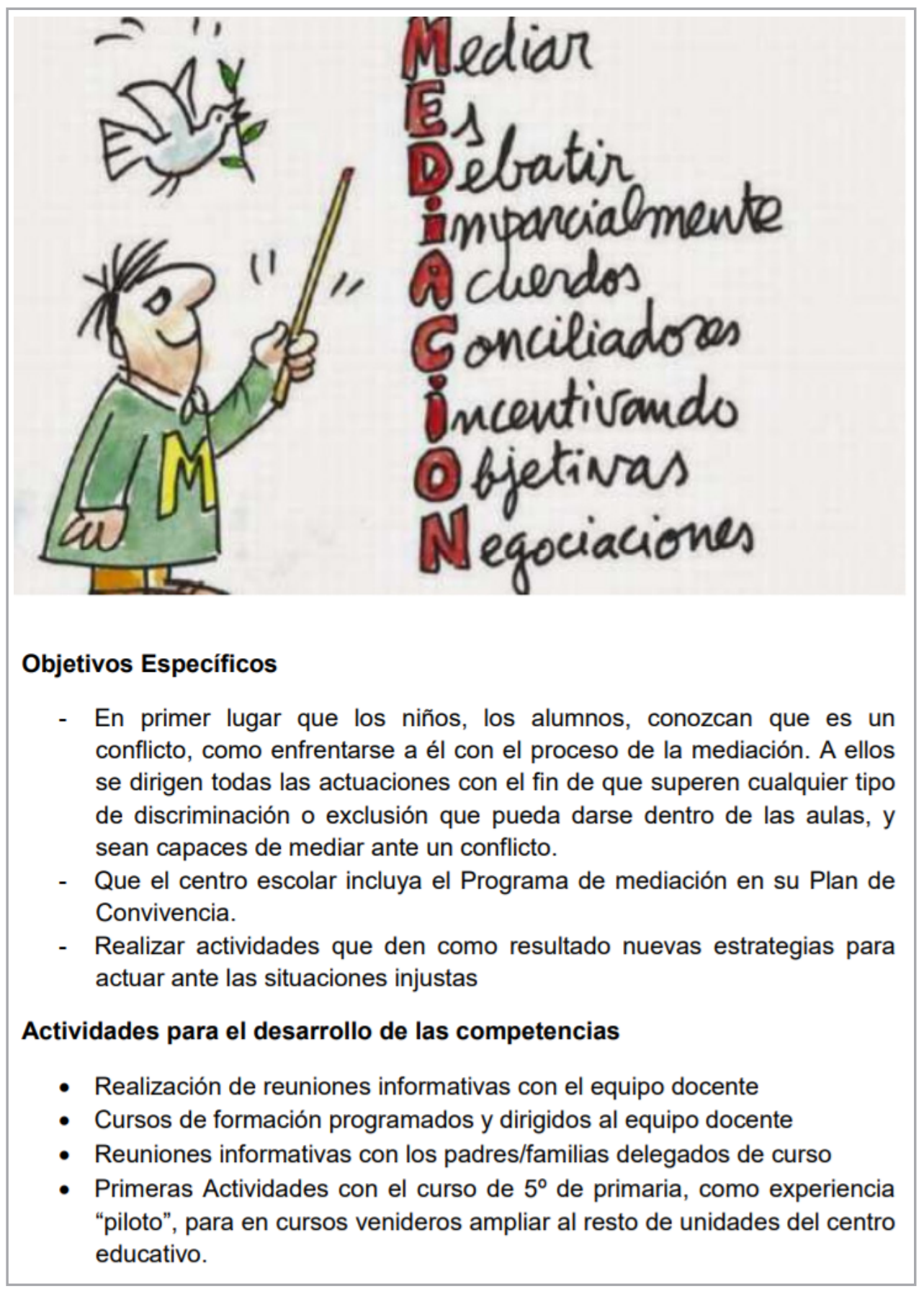

Figura 4. Muestra del trabajo del estudiante sobre diseño de talleres de mediación 


\section{Software educativos para el aprendizaje de Física:}

-ForcePad; programa para visualizar el comportamiento de estructuras sometidas a cargas y condiciones del contorno.

-JMCAD; modela y simula sistemas dinámicos complejos.

\section{Software educativos para el aprendizaje de Química:}

-Jmol; visualizador de estructuras químicas en tres dimensiones.

-BKChem; dibuja sustancias químicas en tres dimensiones y permite explorarlas en diferentes formatos.

\section{Software educativos para el aprendizaje de Geometría:}

-Marble, permite explorar la superficie terrestre y lunar con imágenes de satélites y mapas topográficos, ampliables.

-World Geography Puzzle; presenta lecturas y comentarios de los continentes y océanos del mundo.

Figura 5. Muestra del trabajo del estudiante sobre diseño de talleres de formación en herramientas digitales

Estos ejemplos son solo algunas de las propuestas que los estudiantes han realizado en sus actividades de evaluación final. Como se puede apreciar, las temáticas son diversas y abarcan una grande diversidad de ámbitos de intervención educativa. Las competencias específicas que más se han trabajado son las relacionadas con el diseño de planes, programas y proyectos y con el asesoramiento sobre el sobre el uso pedagógico e integración curricular de los medios didácticos. Asimismo, las actividades de dinamización de foros temáticos virtuales en los que se discute entre alumnos y profesorado y actividades paralelas dinamizadas por el profesorado de aprendizaje informal en Twitter, lo que también confirma similares estrategias didácticas en otras investigaciones (Fernández-Márquez, Vázquez-Cano, y López Meneses, 2016; López-Meneses y Vázquez-Cano, 2013).

En este sentido, compartimos con otros estudios que la innovación es un proceso que debe dar por resultado un producto suficientemente creativo y valioso que enriquezca el bien cultural de una comunidad humana, que en nuestro caso sería la educativa (Atkinson, 2013; Christensen, 1997; López y Heredia, 2017). En este sentido, el análisis de los trabajos de los estudiantes del prácticum ha evidenciado que sus propuestas intentar abordar la innovación educativa como una de las mejores estrategias para mejorar la calidad del sistema educativo (López Meneses, Vázquez-Cano, y Fernández Márquez, 2014; Sevillano y Vázquez-Cano, 2017; Vázquez-Cano, García Iglesias, y Holgueras González, 2018). 


\section{Conclusiones}

La innovación educativa es un requerimiento normativo, pero más que eso, es una necesidad intrínseca a la praxis docente y la organización de la escuela. Nadie cuestiona que un médico o ingeniero tenga que estar continuamente actualizado y que, sin la correspondiente innovación en esas profesiones, la ciencia y la mejora de los procesos y su aplicación a las personas nunca mejoraría. Para mucha gente, incluidos numerosos profesores y padres y madres, la educación de "antes" era la mejor, pero ninguno de ellos se compraría un coche sin "ABS" o sin "airbags" ni se operarían a corazón abierto si lo pueden hacer con una cirugía mínimamente invasiva. La innovación es necesaria en todas las facetas de la vida porque nos hace reflexionar y cambiar prácticas obsoletas o poco eficaces. Por lo tanto, la escuela, formadora y educadora de niños y jóvenes, no puede prescindir de una dinámica que le inste al cambio y a la mejora continua. En esta labor de innovar en la escuela se hace necesario huir de los dogmatismos, buscar las tendencias de innovación holísticas en las que se percibe el cambio como un proceso de ajuste permanente, con pocas certezas y mucho de aprendizaje a través del ensayo-error. Se debe favorecer por parte de los equipos directivos que los docentes más innovadores tengan las condiciones que faciliten su labor y, poco a poco, generar una cultura de centro tendente al cambio y a la experimentación.

Para ello, el profesorado debe buscar nuevas estrategias metodológicas que respondan a estas nuevas demandas. Una adecuada aproximación metodológica es la base de cualquier buena programación; por lo que el docente, deberá tener muy presente para proceder con planteamientos claros y consistentes que demuestren sus habilidades pedagógicas. Algunos de los principios metodológicos fundamentales sobre los que los inspectores de educación pueden asesorar para promover una metodología innovadora se podrían concretar en:

a) Supremacía de la educación sobre la instrucción.

b) Respetar la individualidad del alumno.

c) Atención creciente a los intereses del alumno.

d) Una disciplina basada en la colaboración entre alumnos y profesores, procurando las responsabilidades individuales y sociales.

e) Sustituir competitividad por cooperación.

f) Coeducación, en la que cada sexo ejerza sobre el otro una influencia saludable.

g) Educación que prepare al individuo para ser no solamente ciudadano capaz de cumplir con sus deberes, sino también ser humano consciente de su dignidad de persona.

h) Una educación que posibilite la integración de la persona en sociedad a través de un desarrollo efectivo de la educación en valores y de unas capacidades funcionales por medio de las competencias clave. 
i) Trabajo colaborativo y por proyectos.

j) Nuevos instrumentos para el seguimiento y evaluación del proceso de enseñanza-aprendizaje: procesos de evaluación con base en la aplicación de portafolios, rúbricas, coevaluación y autoevaluación.

k) Integración de metodologías y tecnologías emergentes: clase invertida ("Flipped classroom"), entornos personales interactivos, recursos educativos en abierto, uso de redes sociales de forma educativa, experimentación con realidad aumentada y virtual, apps, programación, robótica, pensamiento computacional, etc. A continuación, mostramos algunos ejemplos de las propuestas de los estudiantes, relacionadas con metodologías activas.

El desarrollo de las prácticas profesionales en Pedagogía desde una mirada participativa y analítica puede contribuir como un agente más de los procesos de enseñanza-aprendizaje a la mejora de las instituciones formativas y educativas desde la identificación de áreas de intervención para la innovación educativa y, en este sentido, también se desarrollan competencias profesionales básicas del pedagogo.

Los procesos educativos en el Prácticum que insten al alumnado a la búsqueda de a innovación en el desarrollo de sus prácticas profesionales, además de contribuir al desarrollo de competencias transversales y específicas, posiciona al estudiante ante el reto de mirar la institución educativa con otros ojos e intentar indagar aquellos procesos susceptibles de mejora y arbitrar propuestas novedosas que persigan la calidad. Asimismo, las metodologías deben ser eminente activas y promover la discusión de las propuestas entre profesorado y alumnado y los tutores de prácticas. Las actividades informales como el uso de redes sociales o microblogging pueden ayudar a visibilizar y debatir sobre propuestas de innovación en el prácticum; ya que permiten un diálogo más fluido y, de esta manera, compartir nuevas miradas sobre los procesos educativos.

\section{REFERENCIAS BIBLIOGRÁFICAS}

Atkinson, R. D. (2013). Competitiveness, Innovation and Productivity: Clearing up the Confusion. The Information Technology \& Innovation Foundation.

Bogdan, R. y Biklen, S. K. (1992). Investigación cualitativa de la educación. Needham Heights, MA: Allyn and Bacon.

Bolívar, A. (2016). Educar Democráticamente para una Ciudadanía Activa. Revista Internacional de Educación para la Justicia Social (RIEJS), 5(1), 69-87. http://doi. org/10.15366/riejs2016.5.1.

Brennan, R. L. y Prediger, D. J. (1981). Coefficient Kappa: Some Uses, Misuses, and Alternatives. Educational and Psychological Measurement, 41, 687-99.

Cabero, J. (2004). Formación del profesorado en TIC. El gran caballo de batalla. Comunicación y Pedagogía. Tecnología y Recursos Didácticos, 195, 27-31. 
Calvo, A. R. y Ballester, F. (2007). Acoso escolar: Procedimientos de Intervención. Madrid. EOS.

Castells, M. y Monge, P. (2011). Network Multidimensionality in the Digital Age. International Journal of Communication, 5, 788-793.

Caverlee, J., Liu, L. y Webb, S. (2010). The Social Trust framework for trusted social information management: Architecture and algorithms. Information Sciences, ScienceDirect, 180, 95-112. http://doi.org/10.1016/j.ins.2009.06.027.

Coll, C., Bustos, A. y Engel, A. (2008). Las comunidades virtuales de aprendizaje. En C. Coll y C. Monereo (Eds.), Psicología de la educación virtual (pp. 299-320). Morata: Madrid.

Christensen, C. (1997). The innovator's dilemma. Boston, Mass.: Harvard Business School Press.

Delors, J. (1996). Los cuatro pilares de la educación. En La educación encierra un tesoro (pp. 91-103). Informe a la UNESCO de la Comisión internacional sobre la educación para el siglo XXI. Madrid: Santillana/UNESCO.

Escamilla, A. (2008). Las competencias básicas. Claves y propuestas para su desarroIlo en los centros. Barcelona: Graó.

European Commission (2007). Key competences for lifelong learning. European Reference Framework. Luxembourg: Office for Official Publications of the European Communities.

Fernández-Márquez, E., Vázquez-Cano, E. y López Meneses, E. (2016). Los mapas conceptuales multimedia en la educación universitaria: recursos para el aprendizaje significativo. Campus Virtuales, 5(1), 10-18.

Fullan, M. (2002). Los nuevos significados del cambio. Barcelona: Octaedro.

Fundación Telefónica (2016). Innovaciones Educativas 2016. Educar para la Sociedad Digital. Disponible en: http://www.fundaciontelefonica.com/.

Garavaglia, A. (2016). Innovation in Education technology: What is the point? Is immersive education the next step? Research on Education and Media, 8(1). http://doi. org/10.1515/rem-2016-0001.

Gimeno-Sacristán, J. (2008). Educar por competencias ¿Qué hay de nuevo? Madrid: Morata.

González Fernández, R. y Medina Rivilla, A. (2017). El desarrollo profesional de los docentes de educación Infantil. En Medina Rivilla, A. et al. (Eds.), Nuevas perspectivas en la formación de profesores (pp. 23-52). Madrid. UNED.

Holsti, O. R. (1969). Content Analysis for the Social Sciences and Humanities. Reading, MA: Addison-Wesley.

King, A. A. y Baatartogtokh, B. (2015). How Useful Is the Theory of Disruptive Innovation? MIT Sloan Management Review, 57(1), 77-90. http://doi.org/10.1017/ CBO9781107415324.004. 
Knoke, D. y Yang, S. (2008). Social Network Analysis. Los Ángeles [etc.]: Sage.

López, C. y Heredia, Y. (2017). Marco de referencia para la evaluación de proyectos de innovación educativa - Guía de Aplicación. 2017. México: Tecnológico de Monterrey.

López-Meneses, E. y Vázquez-Cano, E. (2013). WEB 2.0 Tools for social Educator training in Higher Education. International Journal of Research In Social Sciences, $3(2), 1-13$.

López Meneses, E., Vázquez-Cano, E. y Fernández Márquez, E. (2014). Análisis de la percepción de los alumnos sobre las áreas de intervención del futuro educador y trabajador social a través de una didáctica digital con mapas conceptuales multimedia. RED. Revista de Educación a Distancia, 41, 1-17.

Mendieta, C., Cobos, D. y Vázquez-Cano, E. (2016). La percepción de los docentes sobre la funcionalidad educo-formativa de las TIC en la Universidad Nacional Autónoma de Nicaragua (UNAM-Managua). Revista Latinoamericana de Tecnología Educativa (RELATEC), 15(3), 113-126. http://doi.org/10.17398/1695288X.15.3.113.

Miles, M. B. y Huberman, A. (1994). Qualitative data analysis: an expanded sourcebook. Newbury Park, CA: Sage.

Miranda Martín, E. (2002). La supervisión escolar y el cambio educativo. un modelo de supervisión para la transformación, desarrollo y mejora de los centros. Profesorado, revista de currículum y formación del profesorado, 6(12), 1-15.

Montero, L. (2006). Profesores y profesoras en un mundo cambiante: el papel clave de la formación inicial. Revista de Educación, 340, 66-86.

Naciones Unidas. (2015). Transformar nuestro mundo: la Agenda 2030 para el desarrollo sostenible. A/RES/70/1. Asamblea General.

Narvekar, R. S. (2006). A New Framework to Understand the Technological Innovation Process. Journal of Intellectual Capital, 7(2), 174-186.

Nieto, S. (2006). Razones del profesorado para seguir con entusiasmo. Barcelona: Octaedro.

OECD (2011). Demand-side Innovation Policies. OECD Publishing. http://doi. org/10.1787/9789264098886-en.

OECD (2014). New Insights from TALIS 2013: Teaching and Learning in Primary and Upper Secondary Education. Paris: OECD Publishing.

Rosales López, C. (2012). Contextos de la innovación educativa. Innovación Educativa, 22, 9-21.

Rosales López, C. (2017). Alumnos, maestros, colegios e incidentes en el pensamiento de educadores en formación. Santiago de Compostela: Andavira. 
Santos Rego, M. A., Jover Olmeda, G., Naval, C., Álvarez Castillo, J. L., Vázquez Verdera, V. y Sotelino Losada, A. (2017). Diseño y validación de un cuestionario sobre práctica docente y actitud del profesorado universitario hacia la innovación (CUPAIN). Educación XX1, 20(2), 39-71. http://doi.org/10.5944/educXX1.17806.

Sevillano García, M. L, Pascual Sevillano, M. A. y Bartolomé Crespo, D. (2007). Investigar para innovar. Madrid: Pearson.

Sevillano, M. L. y Vázquez-Cano, E. (2017). Deslocalización de espacios y tiempos formativos universitarios con TIC. Revista de estudios e investigación en Psicología y Educación, 13. https://doi.org/10.17979/reipe.2017.0.13.2106.

Simons, T. (1993). Speech Patterns and the Concept of Utility in Cognitive Maps: The Case of Integrative Bargaining. Academy of Management Journal, 36, 139-56.

Vázquez-Cano, E., Sevillano, M. L. y Méndez, M .A. (2011). Programar en Primaria y Secundaria. Madrid: Pearson.

Vázquez Cano, E. (Coord.) (2017). La inspección y Supervisión de los centros educativos. Madrid: UNED.

Vázquez-Cano, E., García Iglesias, A. M. y Holgueras González, A. I. (2018). La formación en centros de trabajo para los alumnos de formación profesional inicial. Análisis del funcionamiento y perspectivas de futuro. Educar, 54(2), 469-488. https://doi.org/10.5565/rev/educar.867.

Villar Angulo, L. M. (2017). Hojas digitadas en un árbol universitario. Sevilla: autor.

Weingart, L. R., Olekalns, M. y Smith P. (2004). Quantitative Coding of Negotiation Behavior. International Negotiation, 9, 441-55.

Zimmerman, B. J. y Schunk, D. H. (2011). Self-regulated learning and performance. En B. J. Zimmerman y D. H. Schunk (Eds.), Handbook of self-regulation of learning and performance (pp. 1-12). New York: Routledge. 